\title{
Quantitative classification of breast fine needle aspirates using the AxioHOME system
}

\author{
Obiageli NNODU ${ }^{1^{*}}$ and Dulcie COLEMAN ${ }^{2}$ \\ ${ }^{1}$ Department of Haematology, College of Health Sciences University of Abuja, PMB 117, Gwagwalada FCT \\ Abuja, Nigeria. \\ ${ }^{2}$ Department of Histopathology and Cytology, Imperial College of Medicine at Hammersmith Hospital, Du \\ Cane Road, London W12, ONN, United Kingdom. \\ * Corresponding author,Tel: +234-805-7700887,E-mail: obynnodu@yahoo.com
}

\begin{abstract}
Fine needle aspiration cytology is useful in the pre-operative assessment of patients with breast lumps. Lesions are reported as benign, suspicious or malignant. The number of suspicious categories is high in inexperienced hands thus limiting this useful diagnostic tool. The aim was to evaluate quantitative methods of classifying breast fine needle aspirates using the Highly Optimized Microscope Environment system. MayGrumwald Giemsa-stained archived slides were retrieved and smear quality assessed. Fifty epithelial cells corresponding to the cytological grading on each slide were measured using the system's general morphometry program. Generated data was exported to Microsoft Excel for analysis. A significant difference in the mean nuclear area, mean nuclear perimeter, mean largest nuclear diameter was found between the slides graded as benign, suspicious malignant and malignant. C2 \& C4, D 1, P $<0.004$. C4 \& C5, Area P $<0.001$. The means of the number count of nucleoli was able to distinguish between FNAC Benign and Malignant category C2/C5, P $=00440$ and suspicious benign and malignant $\mathrm{C} 3 / \mathrm{C} 5 \mathrm{P}=0.00486$. Morphometry could be useful in situations where experienced cytopathologists are unavailable especially when this program can also measure the degree of dispersion between cells.

(c) 2007 International Formulae Group. All rights reserved.
\end{abstract}

Keywords: Morphometric image analysis, aspiration cytology, breast.

\section{INTRODUCTION}

Breast cancer is the leading malignancy accounting for $16 \%$ and $11 \%$ of all cancer deaths in developed and developing countries respectively with large differences in mortality among countries world wide. (Pisani, 1993; Kestleloot and Zang, 2006).

In 2003, 582,000 people in Africa presented with cancer - 299,000 men and 283,000 women. Current trends as seen on several cancer registries in Ibadan, Calabar, Zaria, and Enugu show increasing relative frequency of cancer of the breast at $25.3 \%$ (Parkin et al., 2003). As these are only hospital figures, the real incidence is likely to be higher due to under reporting with many women dying at home undiagnosed.
Most breast cancers are detected as painless lumps either by the patient or the doctor during a routine physical examination (Kelsey and Ross, 1993). Clinical suspicions of malignancy can be investigated by surgical removal of the lump, i. e surgical biopsy followed by histological examination, by use of large core needle biopsy and by fine needle aspiration cytology. Surgical biopsy is an invasive procedure that requires the use of theatre space and a variable waiting period for processing the sample, but it provides all the required information for a definitive diagnosis, tumour grading and is the gold standard upon which treatment is based. It is however expensive. Large core needle biopsy can be performed in the clinic but it requires 
local anaesthetic and an expensive instrument - the biopsy needle. It however also provides some architectural detail and is processed in the same way as a surgical biopsy sample. Fine needle aspiration of breast lumps can be performed as an out patient procedure with the use of fine needles (Size $23 \mathrm{G}$ or less). It is relatively painless and requires no anaesthetic.

The sensitivity of fine needle aspiration cytology (FNAC) in the diagnosis of breast cancer is $90-95 \%$ in most series (Zarbo et al., 1991). Although the technique is simple, it requires lot of training and practice to acquire and maintain the skill to do it properly. Some women with breast complaints may have benign lesions. The challenge has been to have clearly defined criteria for correctly identifying cases of malignancy while excluding benign processes.

A multidisciplinary approach to the diagnosis of breast lesions seeks to do this. In the "Triple Approach" a combination of clinical examination, mammography /ultrasound and fine needle aspiration are used to evaluate breast lumps (Gray 1996). Definitive treatment can be based on the cytological diagnosis when all three agree i.e. concordance between clinical examination, mammographic and ultrasound assessment. Breast fine needle aspirates are graded cytologically as Benign (C2), Suspicious Benign (C3), Suspicious Malignant (C4), and Malignant (C5) with corresponding categories by clinical examination and by ultrasound.

The success of needle aspiration cytology relies heavily on the ability of the cytologist to identify and characterize cytological changes in the prepared aspirate. This presents a number of problems. Diagnosis is based on visual criteria which are subjective and can be misleading in certain circumstances. The number of visual clues which need to be assessed and the number of options available may limit consistent reproducibility. Cytological classification as C2-C5 in the triple approach is thus subjective with a margin of error. Significant improvement in accuracy occurs with experience (Zarbo et al., 1991). Lesions in the suspicious category require further evaluation by surgical or trucut biopsy. This is not only more expensive but worrying for the patient.

Various quantitative methods have been developed for the measurement of microscopic features in histology and cytology. Most of these are tedious and time consuming to perform and are not employed in routine diagnosis (Beck, 1987; Baak, 1987; Marchevsky and Silage, 1986; Collan et al., 1987; Ellis, 1996). Reliable objective criteria using a simple, readily applicable method can increase the accuracy, efficiency and thus the cost effectiveness of the diagnosis of breast lesions. Objective criteria are not only useful in interlaboratory comparisons but will encourage the widespread use of cytomorphometry to resolve diagnostic problems in breast fine needle aspiration cytology by reducing the error margin during the early learning period before much experience is gained. This is important in our environment where we do not have sufficiently trained manpower in the field.

The objective of this study was to determine if the number of specimens assigned morphologically to the suspicious category $\mathrm{C} 3 / \mathrm{C} 4$ in breast fine needle aspirates can be reduced by employing a quantitative approach using image analysis.

\section{MATERIALS AND METHODS}

This was a retrospective study of archived breast fine needle aspirates diagnosed in the Cytology Department of St Mary's Hospital London.

A search of computer files for breast aspirates graded cytologically as C2, C3, C4 and C5 seen within a two year period (1995/96) was made. The air-dried slides stained by May Grumwald Giemsa according to standard protocol were retrieved from the stored slides and the quality of smears assessed. Only well-stained slides without cracks, containing epithelial cell clusters which were used to determine the cytological grading were selected. 10 slides were selected for each category.

Fifty ductal epithelial cells on areas of minimum nuclear overlap were circled for analysis on each slide using the General Morphometry Programme on the Highly Optimised Microscope Environment (The AxioHOME System) (Brugal et al, 1992). This is a computerized microscope designed to assist pathologists and cytotechnologists in routine clinical tasks. The prototype of the system is an IBM Compatible computer and light microscope in which a built-in high 
resolution computer display image is superimposed on the optical image of the specimen. By using the mouse-driven cursor to click on dialogue boxes and icons, the user can interact with the computer without looking up from the microscope. The image which the HOME system generates is a real optical image. This enables the pathologist to be on familiar ground and the use of the system can take place as part of routine diagnostic practice.

Before each morphometry session, a stage and objective calibration was carried out using the HOME calibration slide. Measurements were made under oil immersion using the small pixel pen on the tool menu to draw around the nuclear margins of the cells and the small mark size to tag the measured cells.

\section{Statistical analysis}

The data generated for each slide was saved and exported in D Base format to Microsoft Excel where descriptive and comparative statistical analysis was made among the categories by means of the Student's t-test using the Microsoft Excel Data Analysis Tool Pak.

The number count of nucleoli on each slide was performed and means determined for each FNAC category. The Student's t-test was also performed among the categories using the means of the number count of nucleoli for each category.

\section{RESULTS}

Forty cases of breast five needle aspirates (FNA) were studied. The slides were from female patients aged 18-85. The mean age was 50 .

Table 1 shows the result of the measurement of nuclear features in each FNA Category presented as means and standard error of the mean. Variables 3-6 describe nuclear size i.e. Area, Perimeter, D1, D2 and variables 7-12 describe nuclear shape i.e. D1/D2, form factor, circularity, ellipticity, regularity, convex hull.

Table 2 is the result of the comparison of the size variables using the Student's t-test. The probabilities giving 9 degrees of freedom are the figures on brackets. The mean largest nuclear diameter (D1) distinguished breast FNA Category C5 from the benign $\mathrm{C} 2$ and suspicious categories C2/C4 (Figure 1).

The mean nuclear area showed some discrimination between the malignant and suspicious categories but not so much between the benign and suspicious categories (Figure 2). Table 3 shows the means of the number count of nucleoli for each FNA Category. Table 4 shows the result of the student t-test of the means of the number count of nucleoli between the categories.

Table 1: Result of the measurement of nuclear features in each fine needle aspirate category.

\begin{tabular}{lcccc}
\hline FNAC Category & C2 & C3 & C4 & C5 \\
\hline $\begin{array}{l}\text { 1. Number of cases (50 epithelial } \\
\text { cell nuclei in each case) }\end{array}$ & \multicolumn{1}{c}{10} & 10 & 10 & 10 \\
$\begin{array}{l}\text { 2. Mean age of cases }(\mathrm{n}=10 \text { for } \\
\text { each category) }\end{array}$ & & & & \\
Variables & 41 & 44.3 & 55.6 & 59.4 \\
3. Area $\left(\mu \mathrm{m}^{2}\right)$ & & & & \\
4. Perimeter $(\mu \mathrm{m})$ & $70.29 \pm 5.148$ & $74.27 \pm 7.648$ & $83.46 \pm 9.832$ & $164.3 \pm 20.82$ \\
5. D1 & $36.66 \pm 1.580$ & $37.38 \pm 2.137$ & $39.09 \pm 2.169$ & $52.69 \pm 3.704$ \\
6. D2 & $10.95 \pm 0.459$ & $11.17 \pm 0.627$ & $11.94 \pm 0.675$ & $16.94 \pm 0.936$ \\
7. D1/D2 & $8.22 \pm 0.376$ & $8.33 \pm 0.526$ & $8.76 \pm 0.559$ & $12.52 \pm 0.772$ \\
8. Formfactor & $1.35 \pm 0.019$ & $1.36 \pm 0.017$ & $1.38 \pm 0.021$ & $1.37 \pm 0.037$ \\
9. Circularity & $1.56 \pm 0.26$ & $1.58 \pm 0.026$ & $1.54 \pm 0.031$ & $1.47 \pm 0.033$ \\
10. Ellipticity & $0.93 \pm 0.077$ & $0.88 \pm 0.006$ & $0.84 \pm 0.008$ & $0.84 \pm 0.011$ \\
11. Regularity & $1.29 \pm 0.58$ & $1.36 \pm 0.017$ & $1.38 \pm 0.021$ & $1.37 \pm 0.037$ \\
12. Convexhull & $7.84 \pm 0.249$ & $7.76 \pm 0.345$ & $7.01 \pm 0.294$ & $7.63 \pm 0.439$ \\
\hline
\end{tabular}

Tabulated values are Means \pm Standard Error of Mean.

Key: Fine needle aspiration cytology category C2= Benign, C3 = Suspicious Benign, C4 = Suspicious Malignant, C5 = Malignant. Variables 3-6 describe nuclear size and variables 7-12 describe nuclear shape. D1 = Largest Nuclear Diameter, D2 = Nuclear Diameter. 


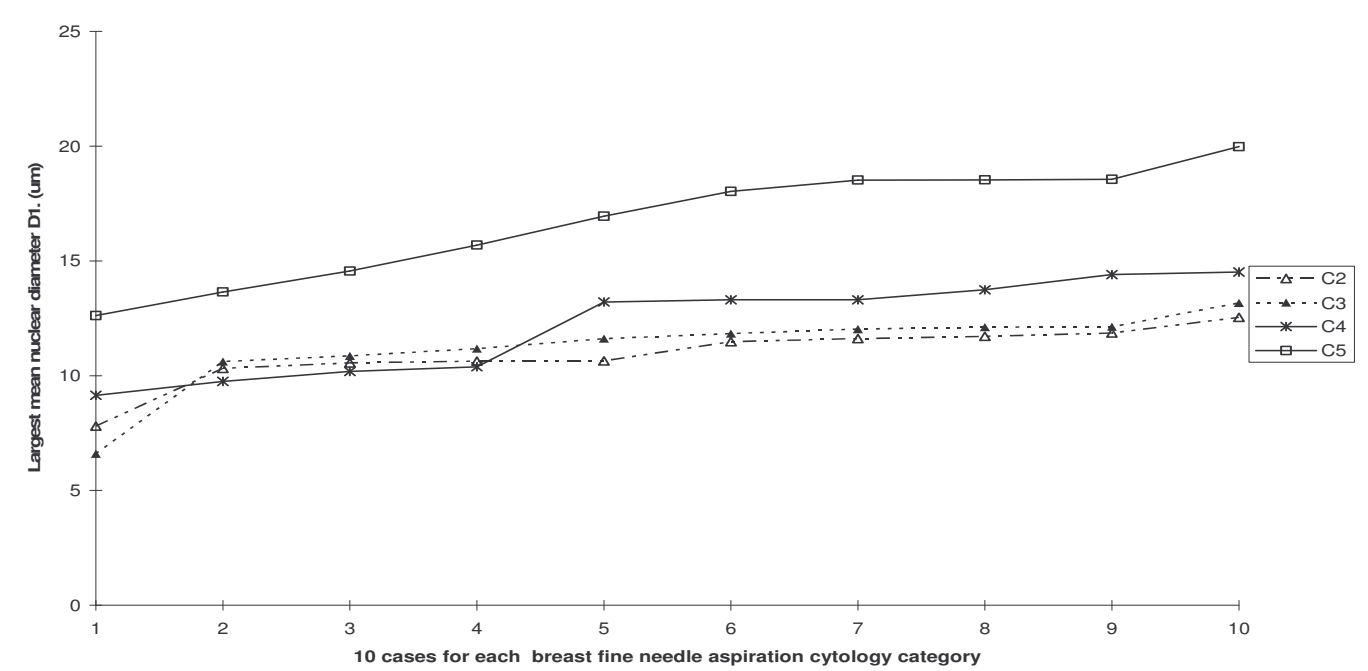

Figure 1: The largest nuclear diameter, D1, for breast fine needle aspiration diagnostic categories. The mean largest nuclear diameter distinguished breast FNA malignant category, C5 from the benign C2 and suspicious categories $\mathrm{C} 3 / \mathrm{C} 4$.

Table 2: Student's t-test analysis of the fine needle aspiration cytology categories.

\begin{tabular}{lccc}
\hline Category & Area & Perimeter & D1 \\
\hline C2 and C3 & 0.8256 & 0.6067 & 0.3914 \\
& $(\mathrm{P}=0.2164)$ & $(\mathrm{p}=0.02498)$ & $(\mathrm{p}=0.2465)$ \\
$\mathrm{C} 2$ and C4 & 2.941 & 3.029 & 3.318 \\
& $(\mathrm{p}=0.008)$ & $(\mathrm{p}=0.007)$ & $(\mathrm{p}=0.004)$ \\
$\mathrm{C} 3$ and C4 & 2.1800 & 1.8371 & 2.4212 \\
& $(\mathrm{p}=0.28)$ & $(\mathrm{p}=0.496)$ & $(\mathrm{p}=0.019)$ \\
$\mathrm{C} 4$ and C5 & 4.2912 & 3.9421 & 4.9661 \\
& $(\mathrm{p}=0.001)$ & $(\mathrm{p}=0.002)$ & $(\mathrm{p}=0.0005)$ \\
\hline \multicolumn{2}{l}{ Key: Fine needle aspiration cytology category $\mathrm{C} 2=$ Benign, $\mathrm{C} 3=$ Suspicious Benign, } \\
C4 = Suspicious Malignant, C5 = Malignant. $\mathrm{D} 1$ = Largest Nuclear Diameter.
\end{tabular}

Table 3: Means of number count of nucleoli for each fine needle aspiration cytology category.

\begin{tabular}{lcc}
\hline Category & Mean & Variance \\
\hline C2 & 4.6 & 16.044 \\
C3 & 5.8 & 48.431 \\
C4 & 13.2 & 136.75 \\
C5 & 21.4 & 182.335 \\
\hline Key: Fine needle aspiration cytology categories- C2= Benign, C3 = Suspicious Benign, \\
C4 = Suspicious Malignant, C5 = Malignant.
\end{tabular}

There was a significant difference between the mean number of nucleoli of $\mathrm{C} 2 / \mathrm{C} 5, \mathrm{P}=$ 0.00440 and $\mathrm{C} 3 / \mathrm{C} 5, \mathrm{P}=00486$.

The means of the number count of nucleoli was able to distinguish between FNAC Benign and Malignant category C2/C5,
$\mathrm{P}=00440$ and suspicious benign and malignant $\mathrm{C} 3 / \mathrm{C} 5 \mathrm{P}=0.00486$. There was no significant difference in the number count of nucleoli between the suspicious benign and suspicious malignant categories. 
Table 4: Result of Student's t-test of the means of number count of nucleoli between the breast fine needle aspiration cytology categories.

\begin{tabular}{lcc}
\hline Paired t-test & Probability & Comment \\
\hline C2/C3 & 0.6674 & Not significant \\
C2/C4 & 0.1426 & Not Significant \\
C2/C5 & 0.00440 & Significant \\
C3/C4 & 0.2470 & Not significant \\
C3/C5 & 0.00486 & Significant \\
C4/C5 & 0.2684 & Not significant \\
\hline Key: Fine needle aspiration cytology categories: C2= Benign, C3 = Suspicious Benign, \\
C4 = Suspicious Malignant, C5 = Malignant.
\end{tabular}

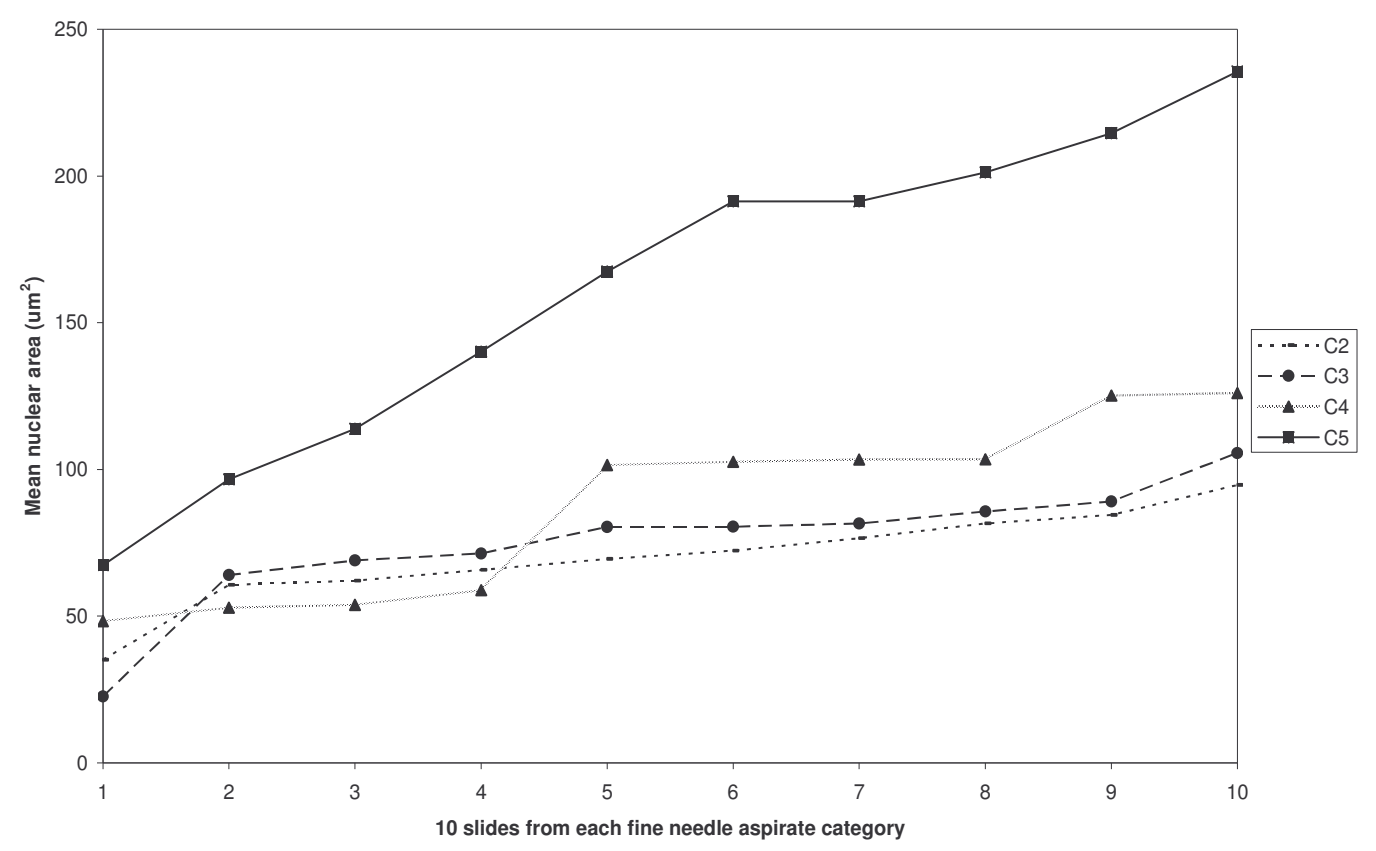

Figure 2: Mean nuclear areas for breast fine needle aspiration diagnostic category. The mean nuclear area showed some discrimination between the malignant and suspicious categories but not so much between the benign and suspicious categories.

\section{DISCUSSION}

At $95 \%$ confidence level, the means of the variables obtained by morphometric image analysis using the HOME System for breast FNA diagnostic categories are as presented in table 2. There was little difference in the means of the shape descriptors such as formfactor circularity, convexity, regularity and convexhull between the FNA categories. The shape variables cannot therefore be used to distinguish one FNA category from the other.

The difference in the largest nuclear diameter, D1, mean nuclear perimeter and the mean nuclear area between FNA Benign, C2, and the suspicious benign C3 categories was not significant. There was however a significant difference in these size variables between C2 \& C4 (Table 3). The t- test for the mean nuclear area was $\mathrm{t}=2.941, \mathrm{P}<0.01$ for the mean nuclear perimeter, $\mathrm{t}=3.3029, \mathrm{P}<$ 0.01, and for the mean largest nuclear diameter $\mathrm{D} 1$, $\mathrm{t}$ was $3.313, \mathrm{P}<0.01$. D1 also demonstrated a significant difference between $\mathrm{C} 3 / \mathrm{C} 4$ and $\mathrm{C} 4 / \mathrm{C} 5$.

However the distinction between the two suspicious categories $\mathrm{C} 3 / \mathrm{C} 4$ was not as marked as that between $\mathrm{C} 2 / \mathrm{C} 4$ and $\mathrm{C} 4 / \mathrm{C} 5$. The mean largest nuclear diameter D1, showed the least standard error of the mean 
and the highest significant level. It is therefore a more reliable and useful criterion than the other size descriptors for this purpose. When combined with the mean of the number count of nucleoli, D1 can be used to distinguish the suspicious benign from malignant categories.

The term "image analysis" is reserved for a special discipline in pathology that aims to obtain diagnostically important information in an objective and reproducible manner by measuring and counting. The introduction of computers has been a major influence on the evolution of image analysis (Meijer et al., 1997). Although studies have shown that nuclear and cellular measurements can be beneficial in the objective diagnosis of breast cytology, the tissue type, choice of texture and methods used to carry out these measurements in previous studies of morphometric analysis of breast lesions have varied widely. Most of these are time consuming and cannot be employed on routine practice. It is therefore not surprising to find a wide range of nuclear sizes quoted in literature for morphometry of breast carcinoma lesions (Walgren et al., 1976; Yu et al., 1995; Wolgerg et al., 1993; Wittekind and Schutte, 1987; Hamilton et al., 1994). Recent studies have also attempted to derive cytoprognostic scores based on nuclear features and nuclear morphometry for breast and prostrate cancer respectively (Fan et al., 2006; Buhmeida et al., 2006). This is the first report of image morphometry on breast fine needle aspirates using the AxioHOME system.

This study has described quantitative features which can be applied in the discrimination of malignant from benign, suspicious benign categories using the mean nuclear area and the mean largest nuclear diameter. The discrimination between the suspicious categories is not that high. Cases with mean nuclear diameter of $16.94 \mathrm{~mm}$, standard error of mean $\pm 0.94 \mathrm{~m}$ can within 95\% confidence limits be said to be malignant. These measurements can easily be carried out using the immediate morphometry program on the HOME System on routine practice.

An extension of this study would be to use the HOME System to ascertain the number of cells on a slide, the degree of cellular dissociation and nuclear pleomorphism and to use this to define morphometric parameters for cytological grading. This will not only have prognostic implications but can be used to follow up patients on adjuvant therapy for breast cancer. Other applications can be carrying out the same measurement on breast FNA Slides prepared by liquid based technology. The system can also be developed to be used in forming part of Grid Technology for information sharing, training, expert opinion, and quality assurance for the remote practitioner by telepathology (Brugal et al., 1992; Gotler et al., 2006).

\section{ACKNOWLEDGEMENTS}

I gratefully acknowledge the help of Robert Baker of the Department of Cytopathology \& Cytogenetics of the Imperial College at St Mary's Hospital, London, for taking me through the initial use of the HOME system as well as the staff of the Statistics Unit and Computer Centre of the same institution for help with the data analysis.

\section{REFERENCES}

Baak JPA. 1987. Principles and advances of quantitative pathology. Anal. Quant. Cytol. Histol., 9(2): 89-95.

Beck JS, Anderson JM. 1987. Quantitative methods as an aid to diagnosis in histopathology. Recent Advances in Histopathology (13th edn). Churchill Livingstone: Edinburgh.

Brugal G, Dye R, Krief B, Chassery J, Tanke H, Tucker J. 1992. HOME: Highly Optimized Microscope Environment. Cytometry, 13: 109-116.

Buhmeida A, Pyrhonen S, Laato M, Collan Y. 2006. Prognostic factors in prostate cancer. Diagnostic Pathology, 1: 4.

Collan Y, Torkkili T, Personen E, Jantunen E, Kosma V. 1987. Application of morphometry in tumour pathology. Anal. Quant. Cytol. Histol., 9(2): 79-87.

Ellis IO.1996. Research techniques and application. In Breast Cytopathology: a Diagnostic Atlas. Chapman and Hall Medical: London; 34.

Fan F, Namiq AS, Tawfik OW, Thomas PA. 2006. Proposed prognostic score for breast carcinoma on fine needle aspiration based on nuclear grade, cellular 
dyscohesion and bare atypical nuclei. Diagn. Cytopathol., 34: 542-546.

Gortler J, Berghoff M, Kayser G, Kayser K. 2006. Grid technology in tissue -based diagnosis: fundamentals and potential developments. Diagnostic Pathology., 1: 23.

Gray W. 1996. Diagnostic Cytopathology. Churchill Livingstone: London.

Hamilton PW, Anderson N, Bartels PH, Thompson D. 1994. Expert system support using Bayesian belief networks in the diagnosis of fine needle aspiration biopsy specimens of the breast. J. Clin. Pathol., 47: 329-336.

Kelsley JL, Horn Ross LP.1993. Breast cancer: Magnitude of the problem and descriptive epidemiology. Epidemiol. Rev., 15: 7-164.

Kestleloot HE, Zahng J. 2006. Differences in breast cancer mortality worldwide: unsolved problems. Eur. J. Cancer Prev., 15(5): 416-423.

Marchevsky A, Gil J, Silage D. 1986. Computerised interactive morphometry as a potentially useful tool for the classification of non- Hodgkin's lymphomas. Cancer, 7: 1544-1549.

McPherson K, Steel CM, Dixon JM. 1994. Breast cancer - epidemiology, risk factors, and genetics - ABC of Breast Diseases. BMJ., 309: 1003.

Meijer GA, Belien J.A.M. van Diest P.J., Baak J.P.A. 1997. Image analysis in clinical pathology. J. Clin. Pathol., 50: 365-370.

Orell SR, Sterret GF, Walters MN, Whitakers D. 1999. Manual and Atlas of Fine
Needle Aspiration Cytology (3rd edn). Churchill Livingstone; 146-152.

Parkin DM, Ferlay J, Hambdi-Sherif, Sitas F, Thomas JO, Wabinga H, Whelan SLDB. 2003. Epidemiology and Prevention. In Cancer in Africa. IARC Scientific Publication no 153: Lyon, France; 11.

Pisani P, Parkin DM, Ferlay J. 1993. Estimates of the world wide mortality from eighteen major cancers in 1985. Implications for prevention and projections for the future burden. Int. J. Cancer. 2; 55 (6): 891-903.

Walgren A, Silfversward C, Zajicek J. 1976. Evaluation of needle aspirates and tissue sections as prognostic factors in mammary carcinoma. Acta Cytol., 20: 313-317.

Wittekind C, Schutte E. 1987. Computerised morphometric image analysis of cytologic nuclear parameters in breast cancer. Analyt. Quant. Cytol. Histol., 9(6): 480484.

Wolgerg WH, Street WN, Mangasarian OL. 1993. Breast cytology diagnosis with digital image analysis. Analyt. Quant. Cytol. Histol. 15: 396- 404.

Yu HG, Sneige N, Kidd LD, Johnston DA, Katz RL. 1995. Image analysis derived morphometric differences in fine-needle aspirates of ductal and lobular breast carcinoma. Analyt. Quant. Cytol. Hist., 17: $88-92$.

Zarbo RJ, Howanitz PG, Bachner P. 1991. Interinstitutional comparison of performance of breast fine needle aspiration cytology: A Q-probe quality indicator study. Arch. Pathol. Lab. Med. 115: $743-750$. 